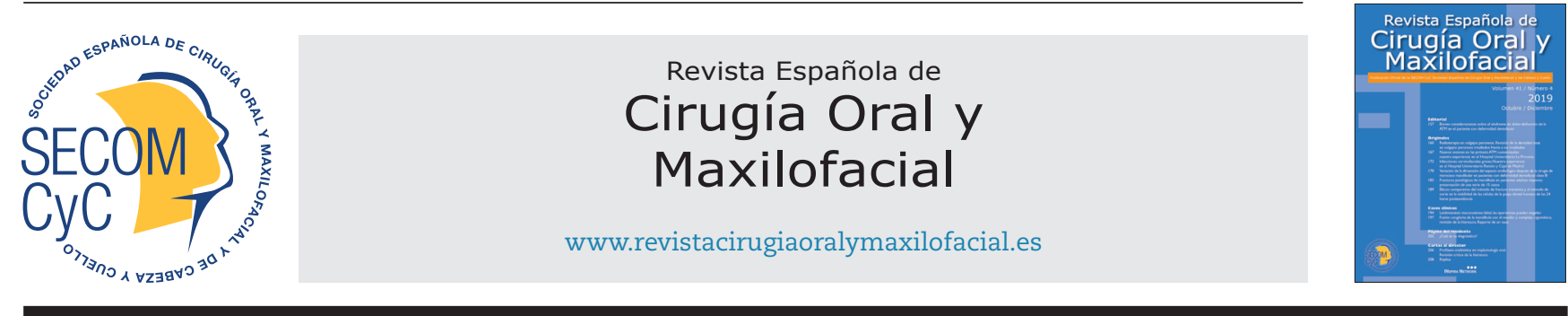

\title{
Original
}

\section{Variación de la dimensión del espacio orofaríngeo después de la cirugía de retroceso mandibular en pacientes con deformidad dentofacial clase III}

\author{
Lizeth Carrión-Mauricio ${ }^{a *}$, Luis Arriola-Guillén ${ }^{b}$ y Hugo Ghersi-Miranda \\ ${ }^{a}$ Magíster Especialista en Cirugía Oral y Maxilofacial. ${ }^{b}$ Magíster Especialista en Ortodoncia y Ortopedia Maxilar, Docente de la Facultad \\ de Estomatología, Universidad Científica del Sur, Lima, Perú. ' Doctor Especialista en Cirugía Oral y Maxilofacial, Docente Principal del \\ Departamento Académico de Medicina y Cirugía Bucomaxilofacial, Facultad de Estomatología Roberto Beltrán, Universidad Peruana \\ Cayetano Heredia, Lima, Perú.
}

INFORMACIÓN DEL ARTÍCULO

Historia del artículo:

Recibido el 5 de enero de 2017

Aceptado el 21 de enero de 2019

\section{Palabras clave:}

Orofaringe, osteotomía, maloclusión de Angle clase III, cirugía ortognática.

\section{R E S U M E N}

Objetivos: Comparar la variación de la dimensión del espacio orofaríngeo (EO) en el prequirúrgico, postquirúrgico inmediato y postquirúrgico de 5 a 24 meses después de la cirugía de retroceso mandibular (CRM).

Materiales y métodos: Se evaluaron las radiografías cefalométricas prequirúrgicas, postquirúrgicas inmediatas y postquirúrgicas de 5 a 24 meses de 20 pacientes con CRM del Servicio de Cirugía Oral y Maxilofacial de dos instituciones. Se cuantificó la dimensión del EO en diferentes momentos.

Resultados: No se encontraron diferencias de significación entre la cantidad de retroceso mandibular y la variación de la dimensión del EO.

Conclusiones: Existe una leve disminución de la dimensión del EO después de la CRM. No son estadísticamente significantes entre los momentos de evaluación. No se encontró correlación entre el retroceso mandibular y la disminución del EO.

${ }^{*}$ Autor para correspondencia:

Correo electrónico: lizkath20@gmail.com (Lizeth Katheryn Carrión Mauricio). 


\section{Variation in the oropharyngeal space after mandibular setback surgery in class III malocclusion patients}

\section{A B S T R A C T}

Keywords:

Oropharynx, osteotomy, malocclusion, Angle class III, orthognathic surgery.
Objective: The aim of this study was to compare the variation in the size of the oropharyngeal space (OS) in the preoperative, immediate postoperative and post-operative 5 to 24 months according to the amount of mandibular setback.

Material and method: Variation were evaluated on cephalometric radiographs taken few days before the surgery (Rx1), immediate postoperative $(\mathrm{Rx} 2)$ and postoperative long time (5 to 24 months) (Rx3). The subjects were 20 patients (18 - 36 years) in whom dentofacial deformity class III was corrected by bilateral sagittal split ramus osteotomy setback. Surgery was done in two Oral and Maxillofacial specialized institutions, since 2003 to 2010. We examined the size of the oropharyngeal space at different times before and after mandibular setback surgery. Results: These shows no significant difference between the amount of mandibular setback and decrease the size of the oropharyngeal space. It was determined that the oropharyngeal space immediately after surgery increases and decreases over time as a result of soft and hard tissue adaptation.

Conclusions: We conclude that there is a slight decreased in the anteroposterior dimension of oropharynx after mandibular setback surgery, however the variations is no statistically significant difference between the moments of evaluation (the amount of mandibular recoil and the decrease in OS).

\section{INTRODUCCIÓN}

Las deformidades dentofaciales clase III son resultado del exceso mandibular, la deficiencia maxilar o ambos ${ }^{1-3}$. El tratamiento quirúrgico con CRM pura es una alternativa de tratamiento, siendo la cirugía bimaxilar más frecuente, por mostrar mejores resultados estéticos y funcionales. Es importante considerar la repercusión de los cambios sobre el tejido blando circundante. Algunos estudios describen la disminución del espacio orofaríngeo (EO) como consecuencia de la CRM, que puede predisponer el desarrollo de desórdenes respiratorios $^{1,3-13} \mathrm{y}$, con ello, contribuir a la aparición del síndrome de apnea-hipopnea del sueño (SAHS), incrementando la morbimortalidad del paciente ${ }^{1,14}$.

El objetivo fue comparar la variación de la dimensión del EO después de la CRM en radiografías cefalométricas de pacientes con deformidades dentofaciales clase III en tres momentos: prequirúrgico, postquirúrgico inmediato y postquirúrgico tardío.

\section{MATERIALES Y MÉTODOS}

Se realizó un estudio observacional, descriptivo, longitudinal y retrospectivo, en radiografías de pacientes del Servicio de Cirugía Oral y Maxilofacial de la Clínica Estomatológica Central de la Universidad Peruana Cayetano Heredia y del Instituto de Cirugía Oral y Maxilofacial (CIROMAX) entre 2003 y 2010, diagnosticados con deformidades dentofaciales clase III, con tratamiento ortodóntico previo, sometidos únicamente a CRM (osteotomía sagital bilateral de rama, técnica de Obwergeser-Dalpont) y fijación con miniplacas y tornillos de titanio. La muestra fue de 20 pacientes de entre 18 y 36 años (prome- dio 23,3 años), 14 mujeres y 6 varones. Se excluyeron radiografías incompletas, pacientes con síndromes y/o deformaciones craneofaciales, antecedentes de traumatismo dentofacial, desórdenes respiratorios o SAHS y antecedentes de alguna cirugía maxilofacial que alterase la morfología o posición de los maxilares.

Se trabajó con radiografías registradas en tres momentos: prequirúrgico de 2 a 4 semanas, postquirúrgico inmediato entre 1 y 2 semanas y postquirúrgico tardío de 5 a 24 meses, tomadas con el paciente en bipedestación, plano sagital paralelo a la placa, oclusión dental, labios y lengua en reposo, y respiración relajada. Las radiografías de la Universidad Peruana Cayetano Heredia se realizaron en un equipo marca Soredex modelo Cranex Tome y las del Instituto CIROMAX en un equipo Planmeca modelo ProMax; en ambos la distancia del ánodo al plano medio sagital del paciente fue de 1,20 m. La magnificación fue de 1,09 (9\%) y de 1,10 (10 \%), respectivamente. El trazado lo realizó uno de los investigadores, previamente calibrado con un especialista en el área. Índice de kappa: coeficiente de correlación intraclase 0,993 e interclase de 0,974 . Se trazaron diez radiografías por día, utilizando el cefalograma de Burstone y Legan ${ }^{15,16}$ para el movimiento mandibular y el de McNamara ${ }^{15}$ para la vía aérea (Figura 1).

Los datos se analizaron con el programa SPSS versión 19. La estadística descriptiva incluyó medidas de tendencia central y dispersión para cada grupo de estudio (retroceso mandibular). Luego se comparó el promedio de cambios de la EO con la prueba de ANOVA, debido a que se transformaron los datos usando el logaritmo natural hasta tener una distribución normal. Las comparaciones múltiples se realizaron con la prueba de Scheffé. Finalmente se utilizó la correlación de Pearson, considerando estadísticamente significativo un valor de $p \leq 0,05$. 


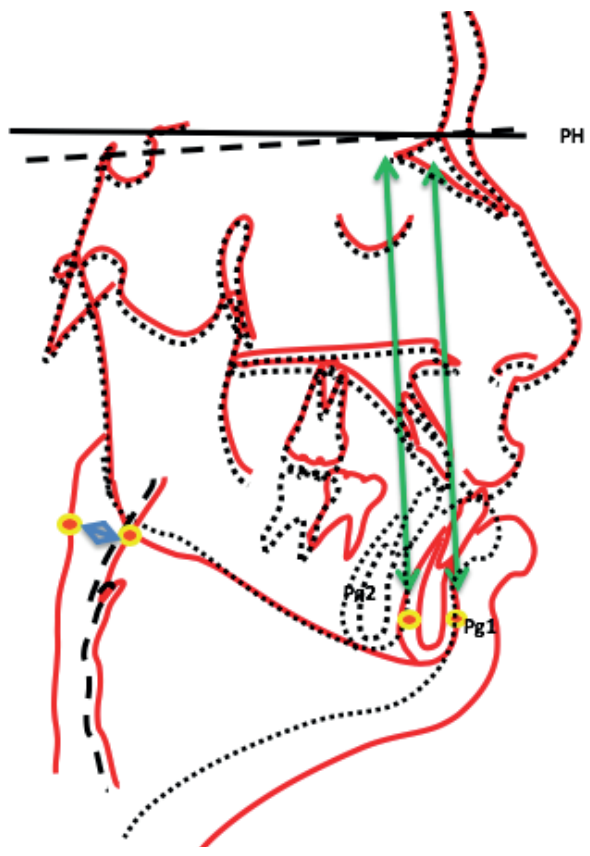

Figura 1. Trazo preoperatorio (línea roja), trazado postoperatorio (línea negra punteada). Pg1: Pogonion en el prequirúrgico. Pg2: Pogonion en el postquirúrgico. Flecha Celeste: vía aérea inferior (orofaringe).

\section{RESULTADOS}

En la Tabla I se muestran las medidas preoperatorias, postoperatorias inmediatas y tardías del EO, pogonion a línea vertical y cantidad de retroceso mandibular. Se encontraron diferencias significativas entre el EO inicial y postquirúrgico inmediato, y entre este último y el postquirúrgico tardío. Al aplicar la prueba de ANOVA, se puede notar que las medias de las medidas de cada variable están en un rango cercano en los diferentes tiempos estudiados, con un descenso en el postquirúrgico inmediato en comparación con los otros dos momentos. La cantidad de retroceso horizontal inicial promedio fue de 3,03 mm. En la Tabla II no se encontró correlación de la variación de la dimensión del EO y el retroceso mandibular. En la Tabla III se muestra la comparación de los cambios del EO según la cantidad de retroceso y los meses de control postquirúrgico: se encontró diferencia significativa en el grupo de pacientes con retroceso mandibular inferior a $6 \mathrm{~mm}$, en contraste con el grupo con retroceso mandibular igual o mayor a $6 \mathrm{~mm}$, donde no se halló diferencia.

\section{DISCUSIÓN}

Los resultados no muestran diferencias estadísticas en la variación de la dimensión del EO después de la CRM. Se encontró que el EO disminuye inicialmente, retornando parcialmente a sus dimensiones iniciales a largo plazo. Los cambios en las dimensiones de la vía aérea tras una CRM están siendo estudiados estas últimas décadas con mayor énfasis; su disminución como consecuencia de una CRM podría ser un factor predisponente al desarrollo de trastornos patológicos, en especial, el SAHS $1,3-11,14,17-21$.

Samman y cols. ${ }^{3}$ compararon el efecto de la cirugía ortognática a los 6 meses en tres grupos: CRM, otro de avance y otro de cirugía bimaxilar; reportaron que solo en la CRM hubo una disminución significativa del espacio orofaríngeo e hipofaríngeo. No encontraron diferencias entre los tres grupos estudiados. Resultados similares hallaron Tselnik y Pogrel ${ }^{14}$, Hochban y cols. ${ }^{9}$, Kim y cols. ${ }^{11}$, Hong y cols. ${ }^{19}$, Achilleos y cols. ${ }^{17}$, Muto y cols. ${ }^{4,13}$, Kawakami y cols. ${ }^{18}$ y Hasebe y cols. ${ }^{19}$. Por su lado, Mattos y cols. ${ }^{20}$ y He y cols. ${ }^{21}$ realizaron un metanálisis y encontraron evidencia de disminución significativa después de la CRM y disminución leve después de una cirugía bimaxilar. Nuestro estudio encontró correlación estadística entre la cantidad de retroceso mandibular y la disminución de la dimensión EO; sin embargo, se halló que en los controles postquirúrgicos tardíos

\begin{tabular}{|c|c|c|c|c|c|c|}
\hline Medida & $\mathrm{n}$ & $\mathrm{X}$ & DS & Mín. & Máx. & $\mathrm{p}$ \\
\hline Orofaringe inicial & 20 & 13,85 & 2,81 & 9 & 18 & \multirow{4}{*}{0,063} \\
\hline Orofaringe PQI & 20 & 13,7 & 3,45 & 8 & 20 & \\
\hline Orofaringe control $\leq 12$ meses & 13 & 11,15 & 3,01 & 7,5 & 18 & \\
\hline Orofaringe control > 12 meses & 7 & 13,93 & 2,34 & 10,5 & 16,5 & \\
\hline PG-LV inicial & 20 & 2,17 & 8,93 & $-16,5$ & 19 & \multirow{4}{*}{0,661} \\
\hline PG-LV PQI & 20 & $-0,85$ & 10,01 & -21 & 18 & \\
\hline PG-LV control $\leq 12$ meses & 13 & $-0,36$ & 7,98 & -19 & 10 & \\
\hline PG-LV control > 12 meses & 7 & $-2,21$ & 12,02 & -13 & 20 & \\
\hline Cantidad de retroceso PG-LV inicial - PQI & 20 & 3,03 & 5,98 & -8 & 13,5 & \multirow{3}{*}{0,936} \\
\hline Cambios PG-LV inicial - PQ $\leq 12$ meses & 13 & 3,52 & 4,66 & -8 & 9 & \\
\hline Cambios PG-LV inicial - PQ > 12 meses & 7 & 2,57 & 6,65 & -6 & 11,5 & \\
\hline
\end{tabular}

Prueba ANOVA. LV: línea vertical. PG: pogonion. PQ: postquirúrgico. PQI: postquirúrgico inmediato. 


\begin{tabular}{lcc}
\multicolumn{2}{c}{ Tabla II. Correlación de los cambios de la orofaringe con la cantidad de retroceso mandibular } \\
Cambios de la orofaringe & Cantidad de retroceso & p \\
\hline Cambios orofaringe inicial - PQI & $-0,144$ & $\mathrm{u}$ \\
Cambios orofaringe inicial - $\mathrm{PQ} \leq 12$ meses & 0,029 & 0,102 \\
Cambios orofaringe inicial - $\mathrm{PQ}>12$ meses & 0,181 & \\
\hline
\end{tabular}

Prueba de correlación de Pearson. PQ: postquirúrgico. PQI: postquirúrgico inmediato.

Tabla III. Comparación de los cambios del espacio orofaríngeo según la cantidad de retroceso y control postquirúrgico $(\mathrm{mm})$

\begin{tabular}{|c|c|c|c|c|c|c|c|c|c|c|}
\hline \multirow{2}{*}{$\begin{array}{l}\text { Cantidad } \\
\quad \text { de } \\
\text { retroceso }\end{array}$} & \multirow[b]{2}{*}{ Medida } & \multirow[b]{2}{*}{$\mathrm{n}$} & \multirow[b]{2}{*}{$\mathrm{X}$} & \multirow[b]{2}{*}{ DS } & \multirow[b]{2}{*}{ Mín. } & \multirow{2}{*}{ Máx. } & \multicolumn{4}{|c|}{ Significación } \\
\hline & & & & & & & $\mathrm{p}$ & $\mathrm{p}(1,2)$ & $p(1,3)$ & $\mathrm{p}(2,3)$ \\
\hline \multirow{3}{*}{$<6 \mathrm{~mm}$} & $\begin{array}{l}\text { 1. Cambios orofaringe } \\
\text { inicial - PQI }\end{array}$ & 12 & $-0,833$ & 2,25 & -4 & 4 & \multirow{3}{*}{0,024} & \multirow{3}{*}{0,024} & \multirow{3}{*}{0,578} & \multirow{3}{*}{0,361} \\
\hline & $\begin{array}{l}\text { 2. Cambios orofaringe } \\
\text { inicial - } P Q \leq 12 \\
\text { meses }\end{array}$ & 7 & $-2,64$ & 1,77 & 0 & 4,5 & & & & \\
\hline & $\begin{array}{l}\text { 3. Cambios orofaringe } \\
\text { inicial - } \mathrm{PQ}>12 \\
\text { meses }\end{array}$ & 5 & 1 & 0,79 & 0 & 2 & & & & \\
\hline \multirow{3}{*}{$\geq 6 \mathrm{~mm}$} & $\begin{array}{l}\text { 1. Cambios orofaringe } \\
\text { inicial - PQI }\end{array}$ & 8 & $-0,5$ & 2,39 & -3 & 4 & \multirow{3}{*}{0,710} & \multirow{3}{*}{0,710} & \multirow{3}{*}{0,960} & \multirow{3}{*}{0,962} \\
\hline & $\begin{array}{l}\text { 2. Cambios orofaringe } \\
\text { inicial - } P Q \leq 12 \\
\text { meses }\end{array}$ & 6 & $-1,5$ & 2,14 & -2 & 4 & & & & \\
\hline & $\begin{array}{l}\text { 3. Cambios orofaringe } \\
\text { inicial - } \mathrm{PQ}>12 \\
\text { meses }\end{array}$ & 2 & 1 & 0,71 & 0,5 & 1,5 & & & & \\
\hline
\end{tabular}

Prueba de Scheffé. PQ: postquirúrgico. PQI: postquirúrgico inmediato.

existe un leve estrechamiento del EO, lo que podría atribuirse a la adaptación progresiva de los tejidos.

Respecto al tipo de imagen utilizada, Meisami y cols. ${ }^{22}$ estudiaron los cambios de la vía aérea mediante resonancia magnética, y concluyeron que la cirugía ortognática no la compromete significativamente. Kawamata y cols. ${ }^{10}$, que realizaron el estudio mediante tomografía computarizada, concluyeron que podría existir un estrechamiento irreversible de la vía aérea después de la CRM. Por su lado, Park y cols. ${ }^{23}$, en tomografías cone beam, encontraron que a los 4,6 meses el EO había disminuido significativamente, recuperándose 12 meses después. Los resultados hallados por nosotros muestran tendencias similares a las descritas, encontrándose disminución del EO en el control postquirúrgico inferior a 12 meses y aumento del EO en controles posteriores al año. Sin embargo, una de nuestras limitaciones fue el uso de radiografías, ya que no se puede evaluar la morfología y los cambios del EO tridimensionalmente, por lo que solo se tomaron medidas horizontales y no verticales. No se debe olvidar que la radiografía es ampliamente utilizada mundialmente por su disponibilidad, accesibilidad, simplicidad y bajo costo. Tampoco se realizó una comparación entre sexos, debido al tamaño de la muestra y su distribución; sin embargo, es importante considerar este aspecto debido a las diferencias fisiológicas entre hombres y mujeres.
Existen evidencias de probables efectos de la CRM como causa del SAHS $3,9,14,24,25$. Tselnik y Pogrel ${ }^{14}$ lo proponen como uno de los factores relacionados. La obesidad también es un factor importante ${ }^{3}$. Chen y cols. ${ }^{1}$, Samman y cols. ${ }^{3}$ y Hoschban y cols. ${ }^{9}$ mencionan que, para la corrección de las deformidades dentofaciales clase III, es preferible la cirugía bimaxilar a una CRM pura, con el fin de prevenir el estrechamiento del EO y que no sea factor predisponente para desarrollar un SAHS. Además, se menciona que los cambios morfológicos en la vía aérea y estructuras faríngeas así como neurológicos son los responsables de esta patología ${ }^{3,26}$. Sin embargo, no se sabe a ciencia cierta si la CRM, como único factor, podría conllevar el inicio de un SAHS. Se deben evaluar otros factores predisponentes como obesidad, cuello corto, macroglosia, úvula larga o excesiva cantidad de tejido blando alrededor del área naso y orofaríngea, con la finalidad de no afectar la función respiratoria del paciente.

Finalmente, no se encontró significación ni correlación entre la cantidad de retroceso mandibular y la disminución del EO, a diferencia de la mayoría de los estudios mencionados anteriormente. La variación en las dimensiones del EO fueron evidentes sin llegar a encontrarse diferencias significativas; no obstante, los resultados ayudarán a entender el comportamiento y adaptación de los tejidos a corto y largo tiempo en las CRM. 


\section{CONCLUSIONES}

La dimensión anteroposterior del espacio orofaríngeo (EO) disminuye levemente después de una CRM; sin embargo, no es estadísticamente significante en los diferentes momentos de evaluación.

No hay correlación ni diferencia del nivel de significación entre los cambios en la dimensión del EO y la cantidad de retroceso mandibular.

\section{AGRADECIMIENTOS}

Los autores agradecen la colaboración y apoyo de las autoridades, profesores y personal administrativo del Servicio de Cirugía Oral y Maxilofacial de la Universidad Peruana Cayetano Heredia y del Instituto CIROMAX, quienes brindaron las facilidades para la recolección de las historias clínicas que se utilizaron en este estudio.

\section{B I B L I O G R A F Í A}

1. Chen F, Terada K, Hua Y, Saitoc I. Effects of bimaxillary surgery and mandibular setback surgery on pharyngeal airway measurements in patients with Class III skeletal deformities. Am J Orthod Dentofacial Orthop. 2007;131(3):372-7. DOI: 10.1016/j. ajodo.2005.06.028.

2. Ellis E, McNamara JA. Components of adult class III malocclusion. J Oral Maxillofac Surg. 1984;42(5):295-305. DOI: 10.1016/0278-2391(84)90109-5.

3. Samman N, Shu S, Xia J. Cephalometric study of the upper airway in surgically corrected class III skeletal deformity. Int J Adult Orthod Orthognath Surg. 2002;17(3):180-90.

4. Muto T, Yamazaki A, Takeda S, Sato Y. Accuracy of predicting the pharyngeal airway space on the cephalogram after mandibular setback surgery. J Oral Maxillofac Surg. 2008;66(6):1099103. DOI: 10.1016/j.joms.2008.01.003.

5. Marsan G, Cura N, Emekli U. Changes in pharyngeal (airway) morphology in Class III Turkish female patients after mandibular setback surgery. J Craniomaxillofac Surg. 2008;36(6):3415. DOI: 10.1016/j.jcms.2008.03.001.

6. Guven O, Sarac U. Changes in Pharyngeal Airway Space and Hyoid Bone Positions After Body Ostectomies and Sagittal Split Ramus Osteotomies. J Craniofac Surg. 2005;16(1):23-30. DOI: 10.1097/00001665-200501000-00007.

7. Chen F, Terada K, Hanada K, Saito I. Predicting the pharyngeal airway space after mandibular setback surgery. J Oral Maxillofac Surg. 2005;63(10):1509-14. DOI: 10.1016/j.joms.2005.06.007.

8. Saitoh K. Long-term changes in pharyngeal airway morphology after mandibular setback surgery. Am J Orthod Dentofacial Orthop. 2004;125:556-61.

9. Hochban R, Schfinnann U, Brandenbttrg R. Mandibular setback for surgical correction of mandibular hyperplasia - does it provoke sleep-related breathing disorders? J Oral Maxillofac Surg. 1996;25(5):333-8. DOI: 10.1016/j.ajodo.2003.04.017.

10. Kawamata E, Fujishita M, Ariji Y, Ariji I. Three-dimensional computed tomographic evaluation of morphologic airway changes after mandibular setback osteotomy for prognathism. Oral Surg Oral Med Oral Pathol Oral Radiol Endod. 2000;89(3):278-87. DOI: 10.1016/s1079-2104(00)70089-8.
11. Kim JS, Kim J, Hong S, Cho J. Pharyngeal airway changes after sagittal split ramus osteotomy of the mandible: a comparison between genders. J Oral Maxillofac Surg. 2010;68(8):1802-6. DOI: 10.1016/j.joms.2009.11.010.

12. Major M, Flores C, Major P. Assesment of lateral cephalometric diagnosis of adenoid hypertrophy and posterior upper airway obstruction: A systematic review. Am J Orthod Dentofacial Orthop. 2006;130(6):700-8. DOI: 10.1016/j.ajodo.2005.05.050.

13. Muto T, Yamazaki A, Takeda S, Sato Y. Effect of bilateral sagittal split ramus osteotomy setback on the soft palate and pharyngeal airway space. Int J Oral Maxillofac Surg. 2008;37(5):419-23. DOI: 10.1016/j.ijom.2007.12.012.

14. Tselnik M, Pogrel A. Assessment of the Pharyngeal Airway space after mandibular setback surgery. J Oral Maxillofac Surg. 2000;58(3):282-5. DOI: 10.1016/s0278-2391(00)90053-3.

15. Zamora C, Duarte S. Atlas de Cefalometría. Bogotá: Amolca; 2003.

16. Burstone CH, James R, Legan H, Murphy G, Norton L, Farmington C. Cephalometrics for orthognathics surgery. J Oral Surg. 1978;36(4):269-77.

17. Achilleos S, Krogstad O, Lybeg T. Surgical mandibular setback and changes in uvuloglossopharyngeal morphology and head posture: a short- and long-term cephalometric study in males. Eur J Orthod. 2000;22(4):383-94. DOI: 10.1093/ejo/22.4.383.

18. Kawakami M, Yamamoto K, Fujimoto M, Ohgi K, Inoue M, Kirita T. Changes in tongue and hyoid positions and posterior airway space following mandibular setback surgery. J CranioMaxillofac Surg. 2005;33(2):107-10.

19. Hong J, Park Y, Kim Y, Hong K, Oh K. Three-dimensional changes in pharyngeal airway in skeletal class III patients undergoing orthognathic surgery. J Oral Maxillofac Surg. 2011;69(11):e401-8. DOI: 10.1016/j.joms.2011.02.011.

20. Mattos C, Vilani G, Sant'Anna E, Ruellas A, Maia L. Effects of orthognathic surgery on oropharyngeal airway: a metaanalysis. Int J Oral Maxillofac Surg. 2011;40(12):1347-56. DOI: 10.1016/j.ijom.2011.06.020.

21. He J, Wang Y, Hu H, Liao Q, Zhang W, Xiang X, et al. Impact on the upper airway space of different types of orthognathic surgery for the correction of skeletal class III malocclusion: A systematic review and meta-analysis. Int J Surg. 2017;38:3140. DOI: 10.1016/j.ijsu.2016.12.033.

22. Meisami T, Keller A, Cooper R, Cameron M, Sándor G. Magnetic resonance imaging assessment of airway status after orthognathic surgery. Oral Surg Oral Med Oral Pathol Oral Radiol Endod. 2007;103(4):458-63. DOI: 10.1016/j. tripleo.2006.07.006.

23. Park S, Kim Y, Son W, Hwang D, Cho B. Cone-beam computed tomography evaluation of short- and long-term airway change and stability after orthognathic surgery in patients with class III skeletal deformities: bimaxilary surgery and mandibular setback surgery. Int J Oral Maxillofac Surg. 2012;41(1):8793. DOI: 10.1016/j.ijom.2011.09.008.

24. Miloro M, Ghali G, Larsen P, Waite P. Peterson's Principles of Oral and Maxilofacial Surgery. $2^{\mathrm{a}}$ ed. London: BC Decker; 2004.

25. Hasebe D, Kobayashi T, Hasegawa M, Iwamoto T, Kato K, Saito C. Changes in oropharyngeal airway and respiratory function during sleep after orthognathic surgery in patients with mandibular prognathism. Int J Oral Maxillofac Surg. 2011;40(6):584-92. DOI: 10.1016/j.ijom.2011.01.011.

26. Eggensperger N, Smolka W, Iizuka T. Long-term changes of hyoid bone position and pharyngeal airway size following mandibular setback by sagittal split ramus osteotomy. J Cranio-Maxillofac Surg. 2005;33(2):111-7. DOI: 10.1016/j. jcms.2004.10.004. 